\title{
Contribution of Sentinel-2 data for applications in vegetation monitoring
}

\author{
Pia Addabbo ${ }^{1}$, Mariano Focareta ${ }^{2}$, Salvo Marcuccio ${ }^{3}$, Claudio Votto ${ }^{3}$, Silvia L. Ullo ${ }^{4}$ \\ ${ }^{1}$ Università Telematica Giustino Fortunato, via Raffaele Delcogliano 12, 82100 Benevento, Italy \\ ${ }^{2}$ Mediterranean Agency for Remote Sensing and Environmental Control (MARSec), via Perlingieri 1, 82100 Benevento, Italy \\ ${ }^{3}$ Università di Pisa, Aerospace Section, Dept. of Civil and Industrial Engineering, via Girolamo Caruso 8, 56122 Pisa, Italy \\ ${ }^{4}$ Università degli Studi del Sannio, Engineering Department, Piazza Roma 21, 82100 Benevento, Italy Portugal
}

\section{ABSTRACT}

With the entry into operation of the Sentinel-2 mission in June 2015, a new land monitoring constellation of twin satellites has been added to the Copernicus project from ESA and new insights have been derived through the combination of Sentinel-2 data with other optical/multispectral data, and with other data from satellites belonging to the same Copernicus project. To this end, the objective of this paper has been to present new added-value tools first through the integration of different satellite platforms: data from NASA Landsat- 8 and ESA Sentinel-1 have been used and combined, and furthermore through the comparison of satellite data all from the same Copernicus project: data from Sentinel- 1 and Sentinel-2 have been jointly processed and compared. Although data from optical/multispectral sensors, as those of Landsat-8 and Sentinel-2, and data from SAR on board of Sentinel-1, are very different, their combination provides useful and interesting results. The integration and combination of these data can find useful application in many fields from oceans to waterways, from land surfaces to fossil deposits, from vegetation to forest areas. In this works authors have focused their interest in green areas and vegetation monitoring applications, by choosing as case of interest the Royal Palace of Caserta and its gardens. The idea has started from the increasing interest in monitoring the cultural heritage monuments and in particular the surrounding vegetation with the green areas and the parks inside. Satellite images can put into evidence boundaries modifications, the vegetation state, their possible degradation, and other phenomena such as changes in the territories due both to natural and to anthropogenic causes. Data combination from different sources as above specified gives a good number of indexes very useful to analyze the vegetation state and its health in a very deep way. Many of these indexes have been calculated and discussed for investigation.

\section{Section: RESEARCH PAPER}

Keywords: remote sensing; Sentinel-1; Sentinel-2; Landsat-8; vegetation monitoring

Citation: Pia Addabbo, Mariano Focareta, Salvo Marcuccio, Claudio Votto, Silvia L. Ullo, Contribution of Sentinel-2 data for applications in vegetation monitoring, Acta IMEKO, vol. 5, no. 2, article 7, September 2016, identifier: IMEKO-ACTA-05 (2016)-02-07

Section Editors: Sabrina Grassini, Politecnico di Torino, Italy; Alfonso Santoriello, Università di Salerno, Italy

Received March 20, 2016; In final form June 22, 2016; Published September 2016

Copyright: (C) 2016 IMEKO. This is an open-access article distributed under the terms of the Creative Commons Attribution 3.0 License, which permits unrestricted use, distribution, and reproduction in any medium, provided the original author and source are credited

Corresponding author: Silvia L. Ullo, e-mail: ullo@unisannio.it

\section{INTRODUCTION}

In the past, capturing images of our planet Earth from space has fascinated people, and it still does. In these days images of the earth are continuously detected and acquired and scientific communities use them to better understand and improve the management of the land itself and its environment [1]. These images allow us to see the world through a wide frame, to witness large scale phenomena, with an accuracy and completeness that human efforts on the ground could barely reach [2]. This becomes extremely beneficial for acquiring data of areas in the world too remote or otherwise inaccessible. The applications range in a wide circle of study subjects, such as geology (i.e. topography or production of DEMs, Digital Elevation Models of the terrains), agriculture (i.e. classification of crops, soil moisture), forestry (i.e. tree biomass, height, species, plantation, deforestation, or forest monitoring and fire detection), cities (i.e. urban structure and density, control for the detection of unauthorized urban construction), natural disaster management (i.e. earthquake monitoring, identification of landslides, damage monitoring and classification), 
oceanography (i.e. waves, wind, ship detection), man-caused disasters management (oil spills and monitoring of patches), to name a few [3]. In very recent years scientists have realized how remote sensing can find a new application by providing accurate, inexpensive, and timely information related to artistic heritage, either natural (parks, landscapes, etc.) or cultural (monuments, archaeological sites, and so on) [4]. The action of man, pollution, corrosion due to rain, cold, harsh weather conditions, solar radiation and thermal stress, can damage the artistic heritage. In addition, operations like excavations and corings have appeared to be risky, expensive and invasive techniques. On the other side, remote sensing presents many advantages: a global vision of the artistic and cultural sites, included the surrounding area; a periodic monitoring of the events, with quite fast intervention in case anomalies or dangers are detected; the identification of certain sites in the world, that present characteristics so special to be classified and associated, for instance, as part of World Heritage; besides the possibility to locate buried, not yet excavated sites. Another consideration is that environmental changes and their actions are very slow and the resulting damages appear only after a very long time. Remote sensing can help also in this sense, because of its ability to discriminate even small changes.

We have underlined in our previous work [5] the peculiarity of remote sensing in providing information not only on the cultural heritage but also on the surrounding vegetation and parks inside. This aspect is very recent, because more extensive research has been performed in the field of artefacts and manufacts, less instead in the field of parks and green areas related to them.

To this end, the objective of this paper is to present new added-value tools by the integration of different satellite platforms: data from NASA Landsat- 8 and ESA Sentinel-1 have been used and combined; and also by the comparison of data from satellites belonging to the same platform, as Sentinel1 and Sentinel-2.

We would like also to highlight that in our previous work as presented in [5] the choice of Landsat- 8 satellite, to use in combination with Sentinel-1, was not made randomly but it was a carefully reasoned choice. In fact at the time of our initial research, Sentinel-2 data were not available yet and the idea to use Lansat- 8 and Sentinel-1 data together was based on two main considerations: the possibility to increase the available information for land and vegetation monitoring thanks to such different data sources, and the opportunity to anticipate results and discussion that could be resumed and confirmed, in a certain manner, as soon as Sentinel-2 would have been available.

In this work our study has focused mainly on green areas in order to analyse and monitoring the health state and the different types of vegetation. Yet, obviously it can be extended to different applications, considering also that we have already carried out a wide research on the use of combined data from Sentinel-1 and Landsat- 8 on other different fields ([5], [6]), by achieving very interesting results that now can be also improved with the availability of Sentinel- 2 data.

The paper is organized as follows: in Section 2, Landsat-8, Sentinel-1 and Sentinel-2 main characteristics are presented and some anticipations on our work given; in Section 3 the area chosen for the analysis is described and the characteristics of data from Landsat-8, Sentinel-1 and Sentinel-2 are discussed for the area of interest; in Section 4 results are provided, through some combinations of different Landsat- 8 and Sentinel- 2 data bands, while Sentinel-1 signals are processed and discussed. In the final Sections, some important indexes are calculated and compared. Results are presented and some conclusions derived.

\section{LANDSAT-8, SENTINEL-1 AND SENTINEL-2}

\subsection{Landsat 8}

The Landsat- 8 (official name: LDCM - Landsat Data Continuity Mission) is the last of the historic Landsat series designed by NASA, one of the first missions of Earth observation and by far the most long-lived with decades of experience from the first satellite in 1972. Launched in February 2013, just a month later the satellite became operational providing shots with a time of 16 days. The improved image quality, compared to the previous Landsat-7, the introduction of new spectral channels and the availability of constant acquisitions make Landsat- 8 a useful tool for monitoring and analysing the territory. It mounts on board the optical sensor OLI (Operational Land Imager) and the thermal sensor TIRS (Thermal InfraRed Sensor). Thanks to their different spectral bands (11 in total) and to their characteristics, it is possible to get satellite maps consolidated as Real Colour Maps, Infrared Maps, Change Detection, Vegetation Indexes, Multiband Fusion with the Panchromatic channel. In addition with the availability of two new bands of the thermal sensor it is possible to obtain maps of temperature with limited noise pollution and production of maps of gravity on land routes from fires. Moreover, the IR (infrared) band 9 allows clouds monitoring in the atmospheric field, while the new Deep Blue band (Band 1) is ideal for the study of coastal zones and phenomena related to aerosol pollution. The mission Landsat- 8 is guaranteed up to 2018 and beyond, thus ensuring stability and comparison in analysis for many decades, with the coaching in the coming years of comparable ESA Sentinel missions. More detailed information can be found in [7].

\subsection{Sentinel 1}

Sentinel-1 constitutes the first series of operational satellites responding to the Earth Observation needs of the EU-ESA Global Monitoring for Environment and Security (GMES) programme. Its design has been driven by the need for continuity of ERS/Envisat class data provision ([8]-[10]). The two-satellite Sentinel-1 constellation carries a Synthetic Aperture Radar (SAR) instrument at C-band and has been designed to address medium and high resolution applications. The SAR operates at a center frequency of $5.405 \mathrm{GHz}$ and has the ability to scan the area with double polarization, that is with support for HH-HV and VV-VH simultaneous co- and crosspolarization receiving channels. Sentinel-1A was expected to be launched in 2013 and its sister Sentinel-1B 18 months later. Actually, the launch of the first satellite, Sentinel-1A, took place on April the $3^{\text {rd }}$ in 2014, and the launch of the second is scheduled to occur by 2016. Four operational modes are supported in terms of resolution and swath width: Strip Map mode (SMM); Interferometric Wide Swath (IWS); Extra-Wide Swath Mode (EWSM) and Wave-Mode (WM). See [11]-[13] for more details.

A Sentinel-1 toolbox is distributed with free license by ESA and is available freely downloaded from [12]. In Figure 1, a screenshot from the Sentinel-1 toolbox is shown, with the different processing that can be launched, as an example. In particular, for the polarimetric elaboration the available PolSARPro package has been used. It is a universal program for 


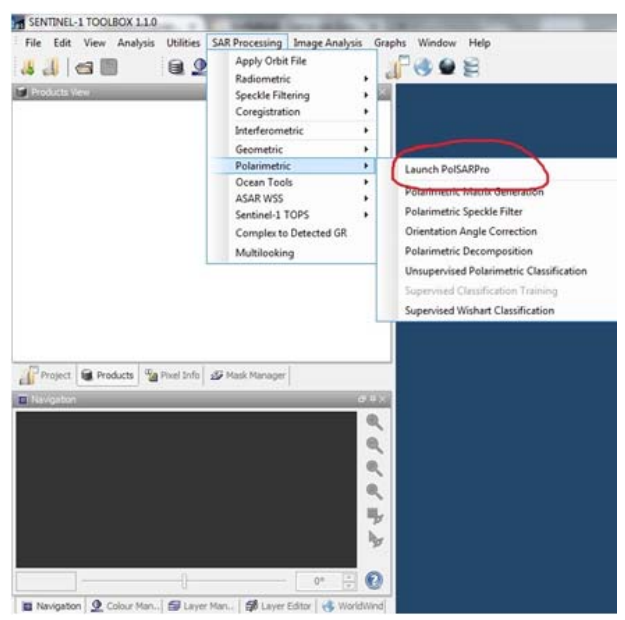

Figure 1. A window of Sentinel-1 toolbox - version 1.1.0.

the management of satellite SAR data, a valuable tool used for any satellite platform. The drawback is that it has many modules and their functionality is wide. Therefore, to get to know all products this software could generate for analysing and processing the Sentinel-1 SAR data, has been a daunting task. Another issue has been that SAR data in order to be compared with other data need to be georeferenced. In effect, both the Sentinel-1 toolbox and the PolSARPro software have the georeferencing function. The problem with PolSARPro was that by dealing only with bursts couldn't process the whole image. Therefore we made the necessary coordinate transformation through a specific QGIS program module. After that, a classification algorithm was used. Among all classification algorithms, we used the Unsupervised Wishart provided by PolSARPro. More precisely, on the basis of a SAR image, we generated a Wishart Supervised classification after an automatic pre-setting with a Wishart Unsupervised. See [11][13] for more details.

As shown in 4.2, different algorithms may be used for SAR image classification. The first technique introduced at this end was the Cloude's decomposition, based on the target coherency matrix and on the decomposition theorem. From Cloude's decomposition, Pauli and Wishart classifications have been then derived. Interested readers can refer to [14]-[18] for more details.

\subsection{Sentinel 2}

The Sentinel-2 mission is a land monitoring constellation of two satellites that provide high resolution optical imagery and provide continuity for the current SPOT and Landsat missions.

It provides a global coverage of the Earth's land surface every 10 days with one satellite and every 5 days with the second satellite, making the data of great use in on-going studies. Sentinel-2A was launched on the 23rd of June 2015 and the first images were available for download in December 2015. Sentinel-2B launch is planned for mid-2016. Sentinel-2A (as Sentinel-2B) is equipped with the state-of-the-art MSI (Multispectral Imager) instrument, that offers high-resolution optical images.

Similarly to Landsat- 8 , Sentinel-2 is provided on board with an optical/multispectral high-resolution sensor, that however operates on 13 different bands of which 4 have a resolution of 10 meters, 6 a resolution of 20 meters and 3 bands a resolution of 60 meters. Therefore, it provides data on the reflectance of the land surface for many different wavelengths, as Landsat- 8 , but for some of them the resolution is much better, until 10 meters versus the 30 meters of Landsat- 8 .

Also in this case, by suitably combining the obtained data, it is possible to get numerous diagnostic and investigative results with geo-referenced information on a local, regional, national and international scale.

ESA distributes free appropriate software for all Sentinel satellite data, as already pointed out in the subsection 2.2. The SNAP (Sentinel Application Platform) is currently available and is programmed for the simultaneous use of data acquired by Sentinel-1, Sentinel-2 and Sentinel-3 satellites, even if the last has been orbited only recently, and its data are not available yet. Having a single software for processing SAR and Optical / Multispectral data turns out to be a huge advantage for their combined use and makes data processing more efficient.

Figure 2 shows a screenshot of the SNAP application, where on the left the command window of the software is open; an acquisition of Sentinel-1 is presented in the middle, where the image (one of three major swaths) is shown as it appears, with the single bursts visible, when the SNAP package is used. Here the processing has been realized at the L1 SLC (Single Look Complex) level, without multilook, therefore it appears "stretched". On the right, an acquisition of Sentinel-2 is shown, in particular an RGB true colour image, obtained by combining the three spectral bands of the visible (Band 4, Band 3 and Band 2).

Figure 2 shows how through SNAP, you can work simultaneously on satellite data of Sentinel-1 and Sentinel-2. In this way, the combined use turns out to be much more practical and simultaneous.

Figure 3 again shows the SNAP software command window together with some pictures of different details of the Royal Palace of Caserta. The first detail on the left represents the

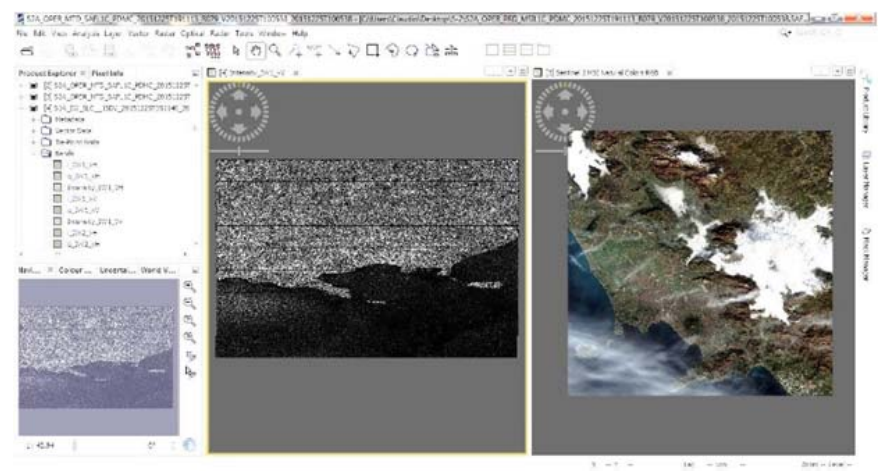

Figure 2. A screenshot of SNAP (Sentinel Application Platform) for Sentinel.

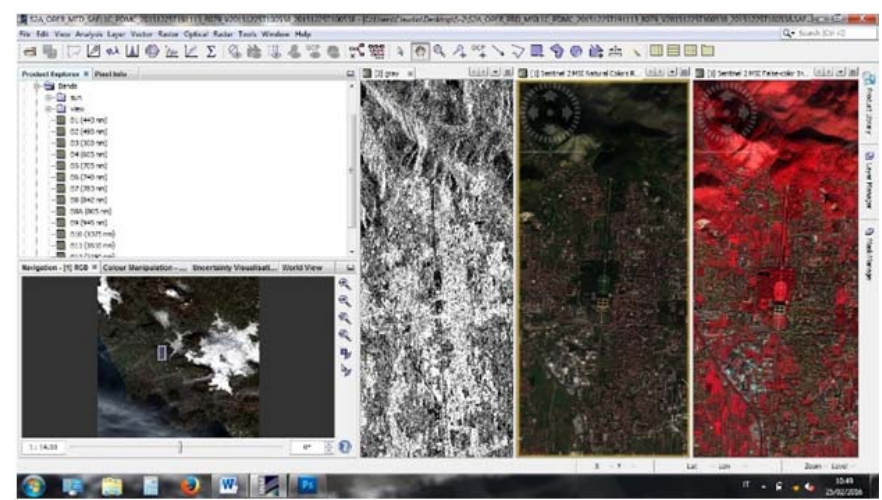

Figure 3. A screenshot of other images obtained with the SNAP application. 
acquisition of Sentinel-1 (a scanned image in IW mode, with VV polarization, not georeferenced); in the middle and on the right there are two RGB images of Sentinel-2, obtained through the combination of the spectral bands in true colours (Bands 4, 3 and 2) and in false colours (Bands 8, 4 and 3) respectively.

It is important to underline that the improvements obtainable now that Sentinel-2 data are available are related not only to the fact that Sentinel-1 and Sentinel-2 satellites are part of the same space mission, and a common platform can be used for processing, but also because Sentinel-2 presents more innovative and advanced features and instrumentation with respect to other satellites that mount on board optical/multispectral sensors as Landsat- 8 for instance.

In Figure 4 the spatial resolution is plotted as a function of wavelength for the different Sentinel-2 spectral bands, by putting into evidence how different bands find suitable applications in different fields of investigation.

As already underlined Sentinel-2 is able to acquire images through 13 spectral bands. Through the main bands (the visible and infrared) it acquires images with a spatial resolution up to 10 meters, differently from Landsat- 8 which reaches only a 30 meter resolution.

Sentinel-2 is also able to acquire images with a spatial resolution of 20 meters through a series of spectral bands in the near infrared (Band 5, Band 6, Band 7 and Band 8a). These bands are very useful especially for the study of agriculture and vegetation. Through their use in fact, it is possible to generate a series of indexes, for instance related to the presence of chlorophyll (which has a very strong response in the infrared), useful for the discrimination and classification of the type of covering present on a territory.

Moreover Sentinel-2 offers three specific bands for the study of cirrus, water vapor and aerosols, whose corresponding images have a resolution of 60 meters. More information can be found at [19].

\section{AREA OF INTEREST AND SELECTED DATA}

The chosen test site was the province of Caserta and included the city of Caserta and the surrounding area for an extension of about 300 square kilometres. The site has been selected by taking into account a number of particular characteristics that could help the combination process between SAR and optical/multispectral data. In particular, we found a little accentuated morphology that could facilitate the observations within the SAR scope, well-defined alternating between urban and predominantly agricultural areas, mountainous areas distinguishable as the different ground covers. These conditions typical of some areas of the Apennines can be optimal for defining parameters and methods

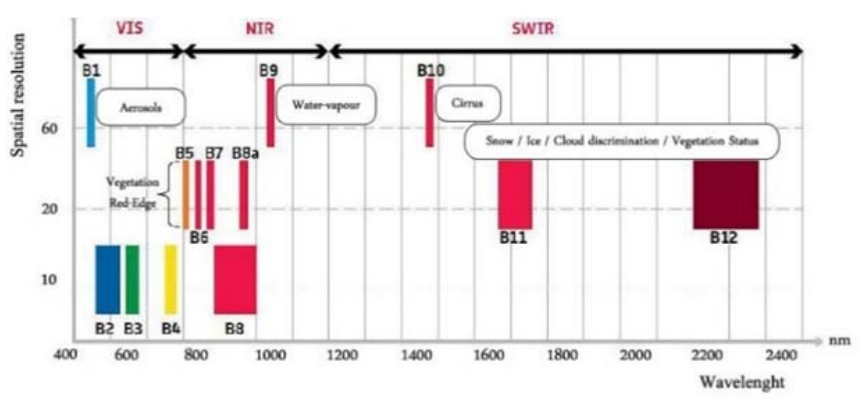

Figure 4. Spatial resolution for the different Sentinel-2 spectral bands, [19]. of analysis that can then be extended to other areas of interest. Given the wide coverage area of single acquisitions by Landsat8, Sentinel-1 and Sentinel-2 satellites, a reduced selected area has been taken into consideration. For instance for Sentinel-1 a single $90 \times 20 \mathrm{~km}$ size Burst has been selected, the Burst $\mathrm{n} .9$ of the second Swath, and the corresponding areas have been chosen from Sentinel-2 and Landsat- 8 images. The covered area is still broad enough to encompass various types of territory: mountain, hilly, urbanized areas, cultivated fields, hedges and wooded grasslands.

To conduct our study we have used Landsat- 8 data downloaded from [20] and Sentinel-1 and Sentinel-2 data downloaded from [21].

In Table 1 all the characteristics for the selected data are summarized.

In Figure 5 we can see how the image from Sentinel-1 (related only to Burst n.9) overlaid a portion of Landsat- 8 image. It should be said that the SNAP software might also be used to process the Landsat- 8 data, but since they are distributed as TIFF images, the QGIS software is better suited for this purpose.

We can observe that the two regions are not completely overlapping since at the left bottom corner there is a small portion not covered. This however has not been a problem because the territory of our interest is all within the selected area. Moreover, we will concentrate our analysis and finalize our conclusions to a portion of the whole area, where there is the Royal Palace of Caserta [22], an artistic and cultural site of big interest. In Figure 6 an aerial photo of the site is shown.

Table 1. Characteristics for Landsat8, Sentinel-1 and Sentinel-2.

\begin{tabular}{llll}
\hline Characteristic & Landsat-8 & Sentinel-1 & Sentinel-2 \\
\hline Acquisition date & Dec. 26, 2015 & Dec. 25, 2015 & Dec.25,2015 \\
Product type & L1T & L1SLC & L1C \\
Acquisition mode & OLI/TIRS & Ascendant/IWS & MSI \\
Information & All bands & Amplitude and phase & All bands \\
\hline
\end{tabular}

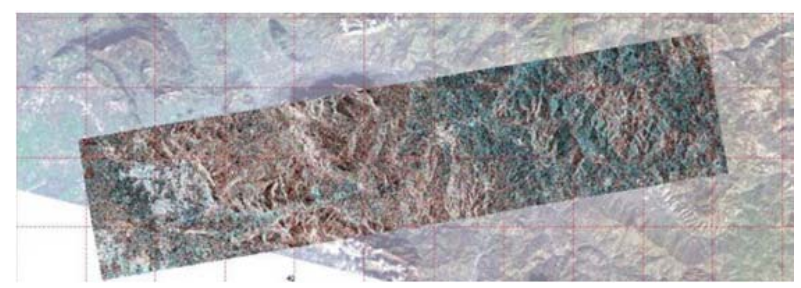

Figure 5. Overlay of Sentinel-1 image (only Burst n.9) and georeferenced Landsat-8 image.

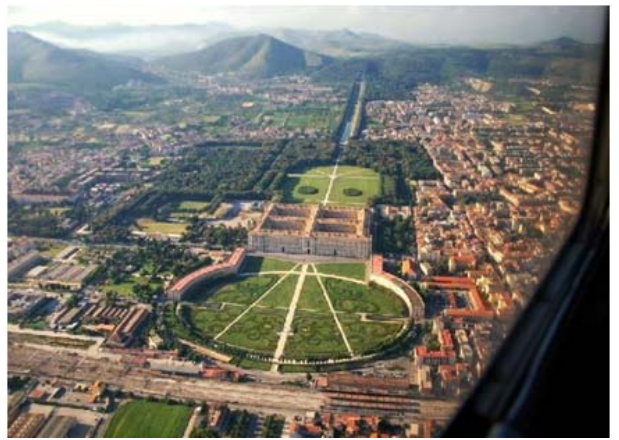

Figure 6. Aerial photo of the Royal Palace of Caserta. 
We can see in Figure 6 that the Royal Palace is adorned with a vast grassland in front and behind the main squared building. Our choice has felt on this site right because of the presence and the huge extension of parks and fountains that have appeared to be suitable to the aims of our study.

In the following Figures 7 and 8, RGB true colour images from Sentinel- 2 and Landsat- 8 are shown.

The difference in the details between the two images is immediately visible, due to the better spatial resolution that Sentinel-2 can reach.

To better appreciate the quality of images reached through Sentinel-2 data, thanks to its high resolution, another image of the same area is shown in Figure 9, where a RGB image of the Royal Palace of Caserta is presented in false colours.

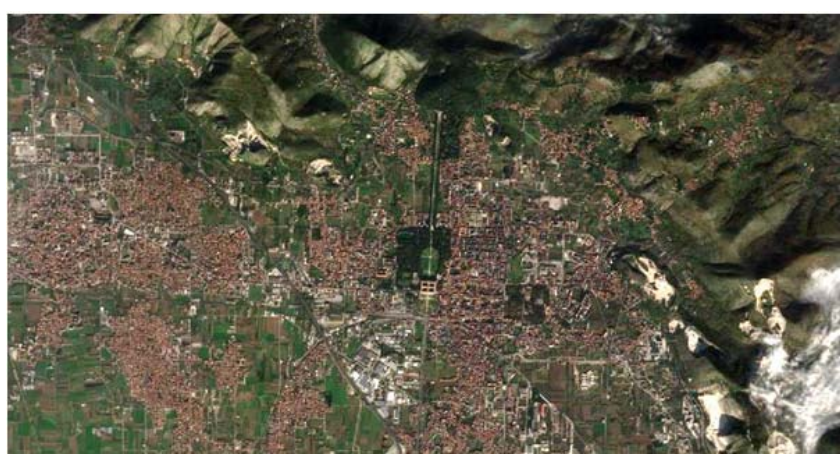

Figure 7. Sentinel-2 RGB true colour image (10 m of resolution).

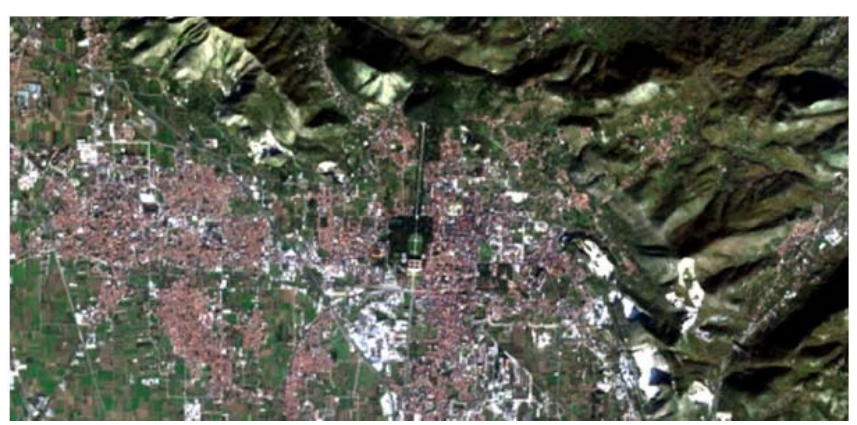

Figure 8. Landsat-8 RGB true colour image (30 m of resolution).

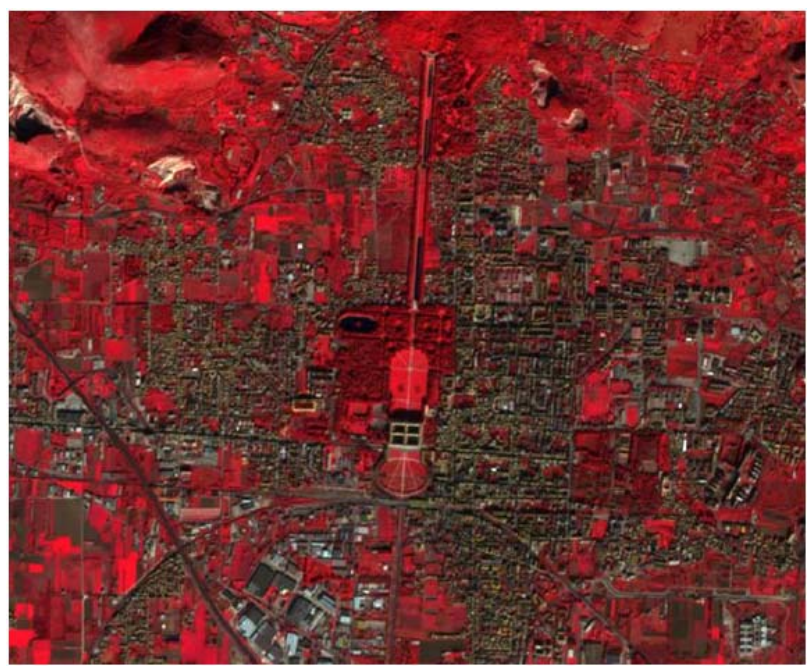

Figure 9. A Sentinel-2 RGB false colour image of the Royal Palace of Caserta (10 $\mathrm{m}$ of resolution).

\section{RESULTS FOR THE SELECTED AREA}

\subsection{Area analysis with Landsat-8 bands combination The NDVI index.}

Landsat- 8 offers eleven bands, some with the OLI sensor, some with TIRS, and Band5 with both. By their combination different images can be plot and several conclusions derived. For sake of simplicity, we will refer to the different combinations just with the corresponding band numbers. In Figure 10 many QGIS images are presented in sequence. The band combination number appears in the bottom corner on the left. From the area of interest a portion including the Royal Palace of Caserta has been selected. In effect, the Royal Palace shape appears almost in the middle of each image, on the right side.

The different bands have been combined in the threechannel RGB (Red, Green, Blue), so as to obtain different unique images in false or true colours, that bring into showing properties of the territory. For instance, the 543 combination corresponds to the image in RGB where in Red is the band 5, in Green the band 4 and in Blue the band 3.

Combination $\mathbf{5 4 3}$ is the standard image in "false colours". The vegetation appears in shades of red, urban areas are cyan and soil varies from dark to light brown. Conifers appear dark red for hardwoods. This combination is useful for studies of vegetation, monitoring of the drainage, terrain models and control of different stages of crop growth. In general, deep shades of red indicate wide leaf and / or healthy vegetation, while the light red tones mean grasslands or sparsely vegetated areas. Urban areas appear in blue. We can see that the Royal Palace appears in blue and all the parks behind and in front of the main central building appear red; it is also possible to see the wooded area in a deep shade of red, surrounding the park on the top. Combination 432 is the image in "true colours". The ground features appear in colours similar to their natural look. Healthy vegetation is green, unhealthy vegetation is brown and yellow; the streets are grey and shorelines are white. Sparsely vegetated or disposed areas are not as easily detected as

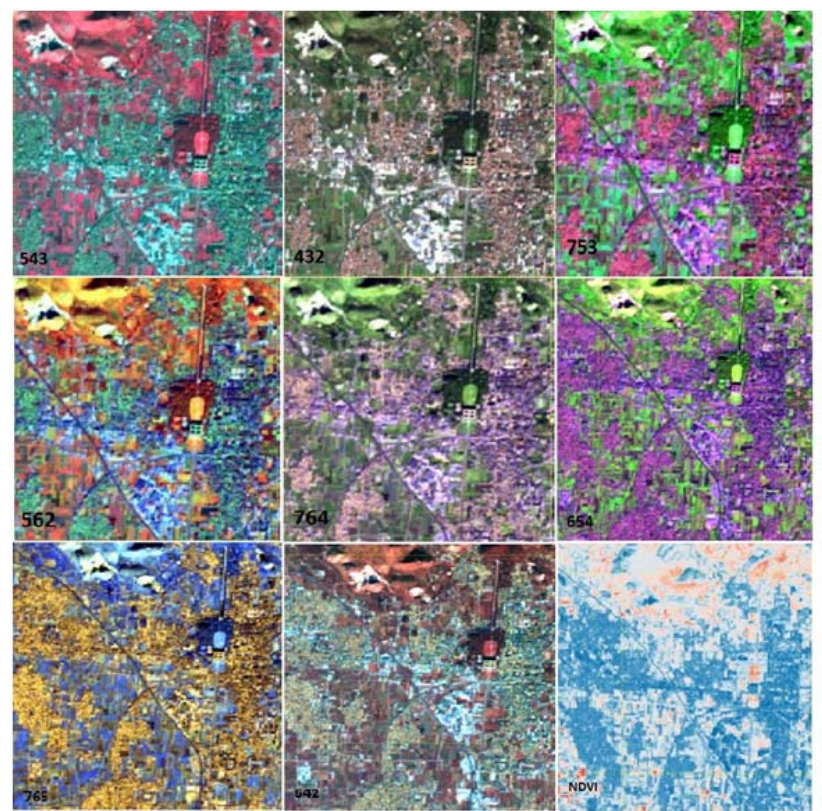

Figure 10. Combinations of Landsat- 8 bands-QGIS Images and the NDVI index on the left bottom corner. 
in 562 or 543 combinations. Moreover also the types of vegetation are not easily distinguishable as in the 562 combination. Shallow waters are not as well distinguished from the soil as in 764 combination. About the Royal Palace, the parks appear in a green colour and the building is light brown. The presence of tanks and fountains is here more evident than in 543. Combination $\mathbf{7 5 3}$ provides a "natural-like" yield. The healthy vegetation appears bright green, grasslands appear green, pink areas represent sterile soil. The orange and brown are for sparsely vegetated areas. Dried vegetation appears orange and the water of blue colour. Sand, soil and minerals are highlighted in a multitude of colours. This band combination provides amazing pictures for the desert regions. It is useful for geological studies, agricultural and wetlands. Urban areas appear in shades of magenta. Grasslands appear bright green and points of green light inside the city indicate a grassy cover, i.e. parks, cemeteries or golf courses. Here the parks of the Royal Palace are very evident with a very bright green colour. In Combination 562 healthy vegetation appears in shades of red, brown, orange and yellow. The deep waters appear to be very dark. Soils can be green and brown; urban characteristics are white, cyan and grey; bright blue areas represent land recently working and reddish areas show new growth of vegetation or grassland probably scattered. For studies of vegetation, the sensitivity to detect various stages of plants growth or stress is increased. In this combination it is possible to differentiate the yellow parks of the Royal Palace from the wooded area surrounding them, that appears very red testifying a wooded area.

Also the Combination 764 provides a "natural-like" yield and also penetrates the weather particles, smoke and haze. The vegetation appears in shades of dark green and bright green during the growth season. Urban characteristics are white, grey, cyan or purple; sand, soil and minerals appear in a variety of colours. Water sources are well highlighted in the image. Flooded areas appear blue or very dark black, much better than the 432 combination where the non-deep flooded regions appear grey and are difficult to distinguish.

Combination 654 as the 562 provides the user with a large amount of information and contrasts. Healthy vegetation is bright green and soils are mauve. While the 753 combination contains geological information, the 654 contains information of a more agricultural nature. This combination is useful for the study of the vegetation and is widely used in the fields of wood management and pest infestation. Combination 765 can be used to study the characteristics of soil weaving and moisture. The vegetation appears blue so if green is preferred this combination should be replaced. This band combination is also useful for geological studies. Combination 642 showcases the topographical features of the land, while replacing the band 7 to 6 , then with the combination 742 , the differences between the types of rocks can be displayed.

In Figure 10 the last image on the left bottom corner refers to the NDVI index. It was generated by combining the near infrared and the visible bands for Landsat- 8 , in order to obtain information on the type of vegetation present on the scene. The index is given by the following equation (1):

NDV I $=\left(B_{5}-B_{4}\right) /\left(B_{5}+B_{4}\right)$.

The NDVI index gives all the information about the status of the crop which are mainly related to the density of biomass produced. For immediate display a scale of colour representation has been chosen, which combines the minimal values to deep blue and the maximum value to deep red. Thus the blue colour indicates areas where vegetation is virtually absent, as buildings, infrastructure and bare lands. Very light blue colours indicate areas where the vegetation is mostly absent or dry. Red colours indicate areas with a certain type of vegetation, that can be poor (very light red) or thick (deep red). Where red is deep, it indicates that the vigour of the vegetation is very high, so it can indicate a plantation in growth or a healthy grassy. Thus the information that is derived from the NDVI index concerns mainly the presence of vegetation cover and its state.

\subsection{Area analysis with Sentinel 1 . The CTest1 index}

After its launch, Sentinel-1A has shown all its great potentiality in terrain observation and high resolution retrievals for getting information on land surface and soil moisture ([8], [23]). A Sentinel-1 SAR image of the area of interest, after a Wishart Supervised Classification, and the image of the same area, processed with the algorithm of Pauli are presented in Figure 11 on the left and on the right, respectively.

It may be noticed that when Wishart is applied, the urbanized area is represented by the red colour while the natural areas are green-yellow. Infrastructures such as roads and railways, and rivers are shown in blue. Furthermore, also the forested areas or in general surfaces with a mainly volumetric geometry are represented in blue. When the same area is processed with the algorithm of Pauli, the image appears a bit different. The polarimetric interferometry module has been used through a Pauli decomposition. The Pauli coherent decomposition provides the interpretation of a full polarimetric SLC (Single Look Complex) data set in terms of elementary scattering mechanism. See [13] and [24] for a full description of the algorithm overview. Through a RGB combination according to the rule of Pauli, the SAR image presents the urbanized area in a light blue colour, whitish for more reflective infrastructures. The land and cultivated fields appear with a colour that ranges from light brown to dark blue, depending on their surface geometry. Mainly flat lands (eg. gravel) are represented by a dark brown. The waterways and the rivers are represented by the black colour. Mountain areas with the side facing the incident direction of the radar appear very bright (white) and with the side in the shade appear very dark (brown almost black).

Similarly to Landsat- 8 , instead of NDVI we have calculated the CTest1 index for Sentinel-1. This index has been obtained by data of Sentinel-1 according to (2):

CTest1 $=\left(\mathrm{S}_{\mathrm{VV}}-\mathrm{S}_{\mathrm{VH}}\right) /\left(\mathrm{S}_{\mathrm{VV}}+\mathrm{S}_{\mathrm{VH}}\right)$.
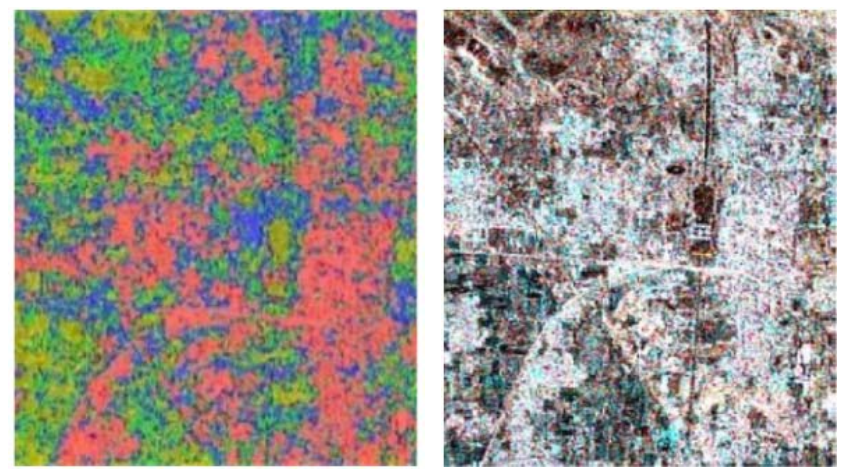

Figure 11. Sentinel-1 SAR images after Supervised Wishart classification (left) and after Pauli rule application (right). 
This equation represents the amount of signal received back by the radar respectively in the two modes: vertical and horizontal polarization. The term $\mathrm{S}_{\mathrm{VV}}$ is representative of the acquisition mode $\mathrm{VV}$, while the term $\mathrm{S}_{\mathrm{VH}}$ is representative of the $\mathrm{VH}$ acquisition mode. The term $\mathrm{S}_{\mathrm{VV}}$ refers to the acquisition mode $\mathrm{VV}$, when a Vertical polarization is used both in the transmitter and in the receiver, while the term $S_{V H}$ refers to the acquisition mode $\mathrm{VH}$, when the signal is transmitted with a Vertical polarization and the receiver is settled on a Horizontal polarization signal acquisition.

The same coloured scale used for the NDVI, has been used also in this case, to facilitate image comparison. The values range from blue colour (minimum) to red (maximum). Related essentially to the geometric characteristics of the surfaces, the signal will be reflected with a horizontal polarization, a vertical polarization, or both. When the CTest1 index is minimum (blue colour), the $\mathrm{VH}$ mechanism reflection is predominant if compared to the $\mathrm{VV}$, that means that the horizontal polarization component in the received signal is very high, while the vertical polarization component is very low or absent.

Therefore, depending on how the signal is received after been reflected from a target, it is possible to understand the type of geometry that has changed the signal polarization. Since the signal is transmitted by the SAR with vertical polarization, the geometry of a target able of returning a signal of this type, must be mainly vertical or close up. On the contrary when horizontal polarization is very high in the received signal, this means that the surface was practically flat. As regarding the vegetated areas, targets associated to this type of geometry are "high" covers, such as vineyards, wheat crops, crops of corn, reeds, etc., in the first case (red colour for the CTest1 index), while areas where blue is predominant indicate that the radar signal has not encountered obstacles that have reflected back the vertically polarized component, but a horizontally polarized signal comes back. Such targets have a geometry predominantly "flat"; they are surfaces which dissipate most of the signal sent from the satellite. The targets associated to this type of response are "low" coverages, such as grassy fields, joke grounds, young plantations, and so on.

\subsection{Area analysis with Sentinel 2. The NDVI index.}

Similarly to the case presented in 4.1, the NDVI index can be calculated also for Sentinel-2, by obtaining information about the state of the vegetation in the area of interest. The only difference is that for Sentinel-2 the near infrared band is the Band 8. The formula for NDVI index in this case is the following, given by (3):

$\mathrm{NDV} \mathrm{I}=\left(\mathrm{B}_{8}-\mathrm{B}_{4}\right) /\left(\mathrm{B}_{8}+\mathrm{B}_{4}\right)$.

Thus the two indexes present the same visible green band, the Band 4, for both, while they differ in the near infrared band ( $\mathrm{B}_{5}$ for Landsat -8 and $\mathrm{B}_{8}$ for Sentinel-2).

In Figures 12 and 13, the two indexes are shown referring to the area around the park of the Royal Palace.

The more precise definition of the details given by Sentinel-2 is clearly evident, thanks to its better resolution that makes the NDVI index very useful also for the study of very small areas.

In Figure 14 a detail of the park (approximately $600 \times 300$ meters) has been taken inside the Royal Palace for comparison between Sentinel-2 and Landsat- 8 satellites.

To see how different their acquisitions are, the NDVI index for both has been calculated and compared for the selected area shown in Figure 14, where vegetation is predominant, see Figure 15.
It is possible to appreciate the big difference between the two indexes due to different resolutions for Sentinel-2 and Landsat- 8 that result in different pixel dimensions.

\subsection{Statistics of NDVI index for Sentinel 2 and Landsat-8}

Figure 16 shows the histograms for the distribution of the analyzed pixels for the NDVI indexes in the two cases. The

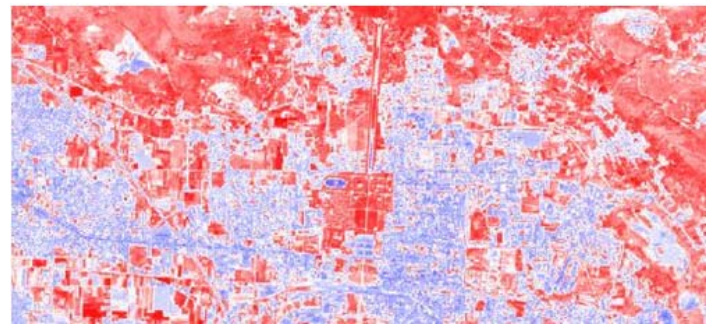

Figure 12. Sentinel-2 NDVI Index.

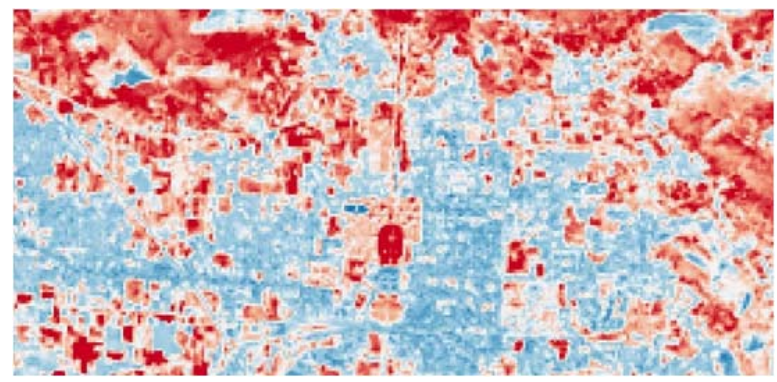

Figure 13. Landsat-8 NDVI Index.

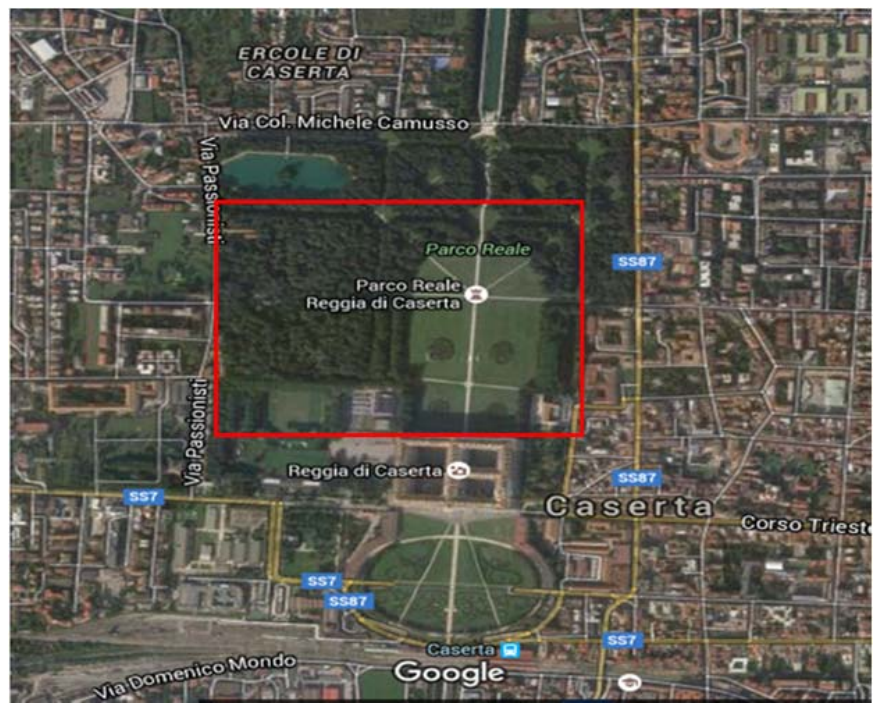

Figure 14. A detail of the park inside the Royal Palace of Caserta.

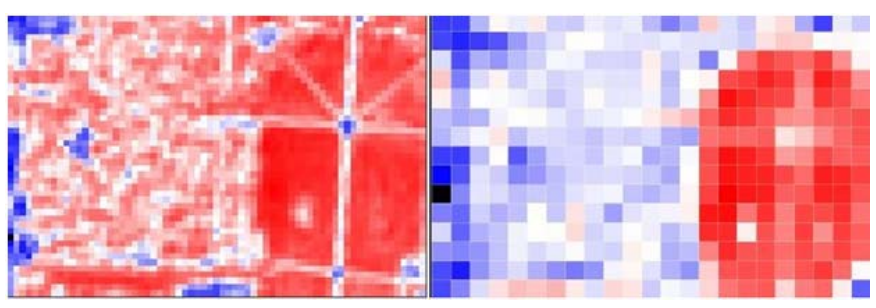

Figure 15. NDVI for Sentinel-2 (left) and Landsat-8 (right) on the selected very vegetated area. 
SNAP application allows to process data and calculate also the statistics for specific indexes and parameters of interest. Figure 16 shows the screenshot of this elaboration.

Thanks to the higher resolution, in the same area NDVI index for Sentinel-2 samples 3015 pixels with respect to the 345 pixels sampled by the NDVI index of Landsat- 8 .

If we look at the statistics, the analysis shows a standard deviation in the acquisition of Landsat-8 $(\sigma=0.0785)$ lower if compared to that of Sentinel-2 $(\sigma=0.1262)$, which apparently would seem to show a minor deviation from the mean value for Landsat- 8 , and therefore a more accurate analysis. In effect, by comparing the variation coefficients, or the dispersion indexes, which allow to compare measurements of phenomena relating to different units of measurement, it emerges that for the acquisition of Landsat- 8 there is a greater variability (CoefVariation $=0.68)$ and therefore a greater dispersion with respect to the acquisition of Sentinel-2 (CoefVariation $=0.41)$.

Moreover, the maximum value that can be measured by Sentinel-2 appears to be more than twice of that measured by Landsat-8.

All this demonstrates the improved efficiency of Sentinel-2 when compared to Landsat-8.

Another very important advantage that can be gained by using Sentinel-2 data, is given by the possibility of using the images captured through the spectral bands in the near infrared, which can generate a series of indexes for the vegetation analysis that cannot be obtained through Landsat- 8 data. Refer to [25] for more information about all the possible indexes that can be calculated.

\section{INDEXES CALCULATION AND RESULTS COMPARISON}

To define and classify the type of coverage present on the ground and show the results of our analysis, we have compared the information obtained from the NDVI index with those obtained from the CTest1. We have further restricted the area of interest by taking into consideration just a part of the zone around the Royal Palace of Caserta.

As shown in Figure 17 the NDVI index on the left identifies the central building with a deep blue colour, while grassland appears in red. The index gives also information about the vigour of the vegetation, that is high (brilliant red), but does not
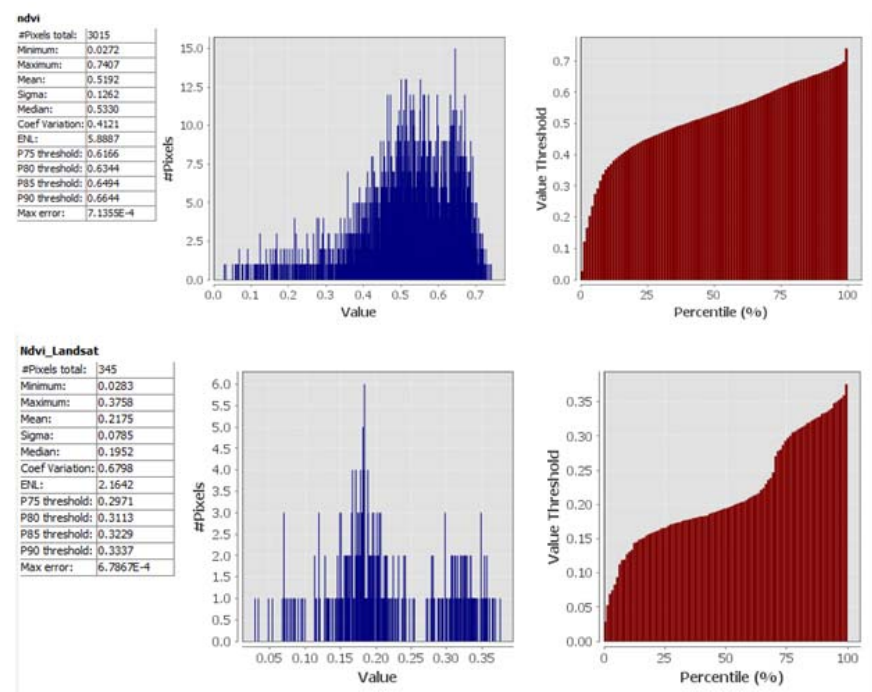

Figure 16. Statistics for Sentinel-2 (top) and Landsat-8 (bottom) NDVI index. provide other information for instance on the type of coverage. Through the index Ctest1, represented on the right of Figure 17, the building is identified with a light pink colour and the ground grass with a deep blue colour. This indicates that from the building the signal is sent back to the radar through a double bounce, thus the horizontal and vertical components of the signal are almost equal, typical response of buildings; while the response on grasslands highlights how the signal sent back to the radar disperses almost completely and the satellite can capture back only a horizontal component sent from the reflection on the ground. This is clearly indicative of the fact that the target has a predominantly flat geometry.

If we put together this information to that given by NDVI (vegetation of high vigour) we can conclude that the place corresponds to a healthy grassy field, just as the photo of the Royal Palace shown in Figure 6 confirms. Data comparison and combination show that only putting together the results obtained from these indexes the final information is complete and helpful. Moreover, we can observe that the thin strip of water formed by the tanks in the garden of the Palace appears blue both with the NDVI index and with the Ctest1. This means that the area is without vegetation and flat, i.e. a water mirror. In practice, the NDVI index can tell us how vegetation is: dry or healthy; and the CTest1 index tells more about geometry: flat or rugged profiles, tall or small targets. In the case of The Royal Palace the two indexes together say: "grasslands healthy, well maintained".

The availability now of Sentinel-2 data with respect to our previous work [5], allows to extend our analysis and to obtain more interesting results.

As already mentioned, in addition to acquiring images through the main band in the near infrared (Band 8), Sentinel-2 has also other spectral bands in the near infrared through which acquire other images. Figure 18 shows the position of these spectral bands for Sentinel-2 according to their centre wavelengths, together with the reflectance response of healthy (green) vegetation, dry vegetation and soil as a function of the wavelength itself [26].

Many studies have been conducted to investigate the relationship between the biological status of plants and their spectral responses since Chlorophyll (Chl), as well as a number of other pigments, are strong absorbers of light in certain welldefined wavelengths, specifically in the blue and the red spectral regions. Pinar and Curran [27], and Filella and Peñuelas [28] found that the Red Edge region is sensitive to $\mathrm{Chl}$ content and $\mathrm{N}$-status. These studies revealed that if $\mathrm{Chl}$ concentration increases, the typical slope in the Red Edge spectral region

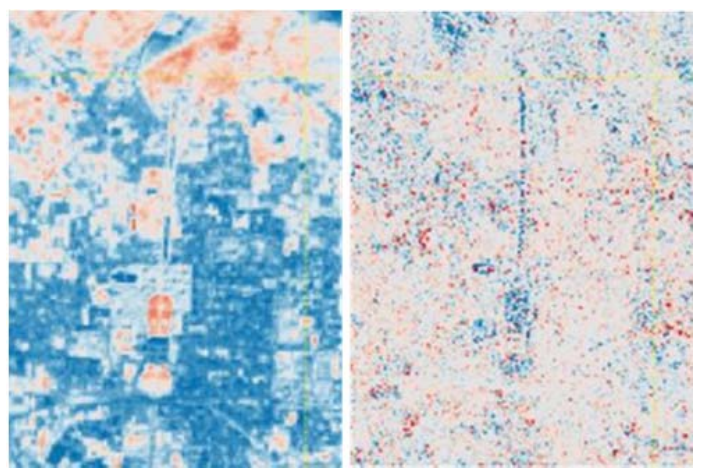

Figure 17. Comparison between Landsat-8 NDVI and Sentinel-1 CTest1 indexes. 


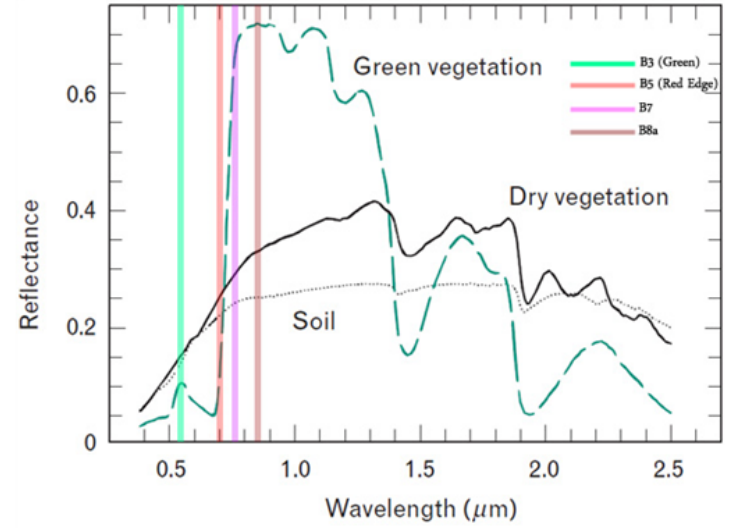

Figure 18. Reflectance of green, dry vegetation and soil with Sentinel-2 bands visualization [26].

shifts towards the Near-Infrared (NIR). Since the Red Edge band is sensitive to Chl status and leaf and canopy structure, it is expected that this band would contribute to the characterization of different plant cover types [29].

For example, Figures 19 and 20 show the images obtained respectively for the Green Chlorophyll Index (Cl_Green) and the Red Edge Chlorophill Index (Cl_Red Edge) of the Royal Palace park. They are two of the most important indexes known in the literature for vegetation monitoring [30].

They are defined by (4) and (5):

$\mathrm{Cl}_{\text {Green }}=\frac{\mathrm{B}_{7}}{\mathrm{~B}_{3}}-1$,

$\mathrm{Cl}_{\text {Red Edge }}=\frac{\mathrm{B}_{7}}{\mathrm{~B}_{5}}-1$,

where spectral Band 3, Band 5 and Band 7 of Sentinel-2 are used for calculations.

The Cl_Green is an index that shows and distinguishes green areas in the territory.

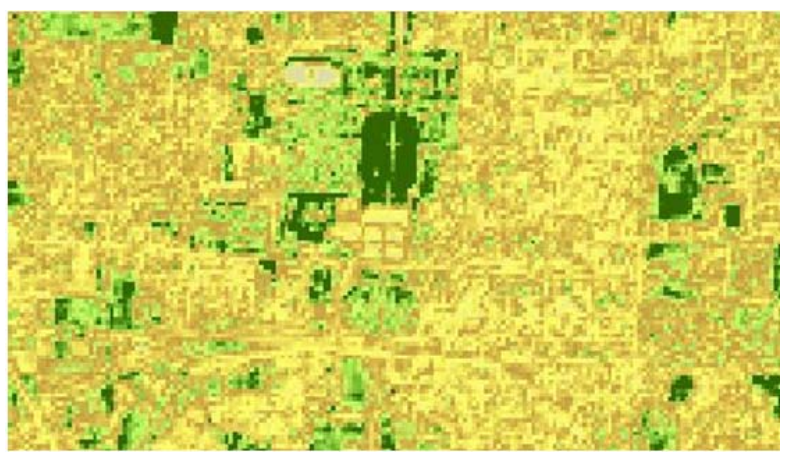

Figure 19. Sentinel-2 Cl-Green Index.

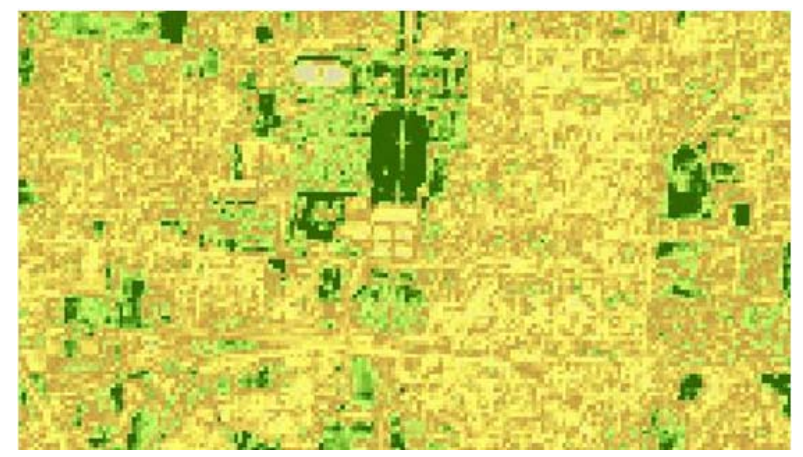

Figure 20. Sentinel-2 Cl-Red Edge Index.
The Cl_Red Edge is an index of the amount of chlorophyll present in the analysed territory.

Both images were represented in false colours where the minimum values correspond to a brownish almost yellow colour that tends to a bright green for the maximum values.

If we furtherly restrict the area under analysis inside the park of The Royal Palace of Caserta, as shown in Figure 21, which refers to a $250 \times 300$ meter surface, we can calculate the indexes over a completely vegetated area for a specific analysis. In order to compare the data of Sentinel- 2 and Landsat-8, even if the images have different resolutions, we have used the main bands in the near infrared, which are comparable. The indexes expressed in (6) and (7) have been calculated:

RECI_Cl $l_{\text {green_}} 2=\frac{\mathrm{B}_{8}}{\mathrm{~B}_{3}}-1$ (Sentinel-2),

$\mathrm{Cl}_{\text {Green_Landsat }}-8=\frac{\mathrm{B}_{5}}{\mathrm{~B}_{3}}-1$ (Landsat- 8 ) .

In (6) and (7) the Band 8 and the Band 5 are respectively the infrared band of Sentinel-2 and Landsat- 8 , while Band 3 is for both the spectral band in the visible green.

In Figure 22 the statistics for the indexes calculated for Sentinel-2 and Landsat- 8 with (6) and (7) are represented. The SNAP application has been used. The results show a clear difference in the maximum values measured by the two satellites (3.07 with Sentinel-2) and (0.99 with Landsat-8).

Analysing the same area through the index Cl_Green (Green Chlorophyll Index), here renamed RECI_Cl_Green, defined by the (8):

$$
\text { RECI_Cl }{ }_{\text {Green }}=\frac{\mathrm{B}_{7}}{\mathrm{~B}_{3}}-1
$$

and obtainable only for Sentinel-2, the results turn out to be better than the index RECI_Cl_green_2 of (6).

In fact, thanks to the fact that the Band 7 of Sentinel- 2 is much narrower $(20 \mathrm{~nm})$ compared to the Band $8(115 \mathrm{~nm})$ used in the index RECI_Cl_green_2, the measurement is much more sensitive, and obtained values have higher highs $(\max =3.166)$ as shown in the Figure 23.

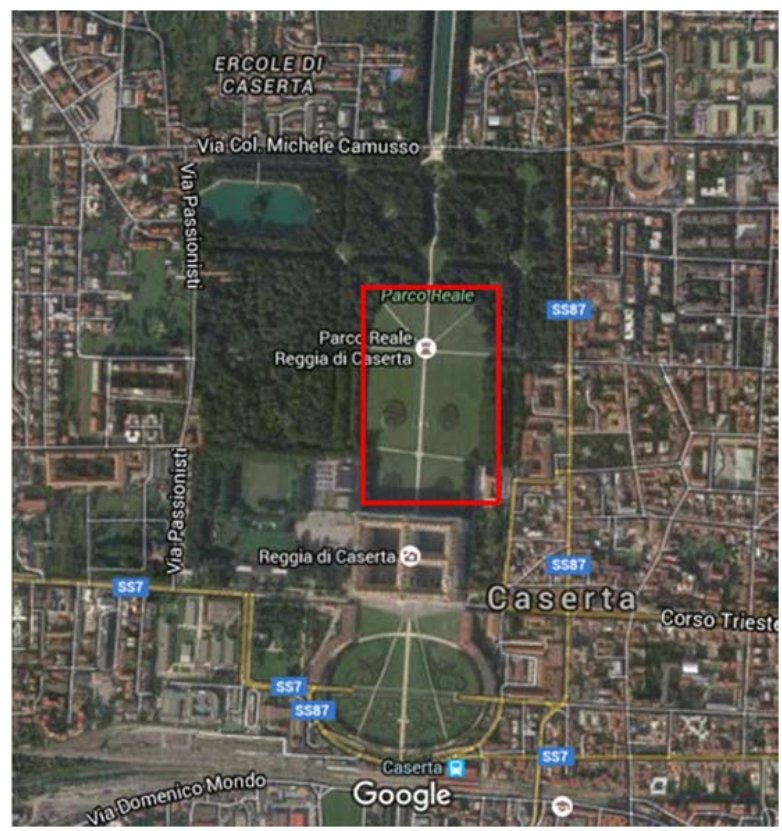

Figure 21. A $250 \times 300$ meter area selected inside the park of the Royal Palace of Caserta. 

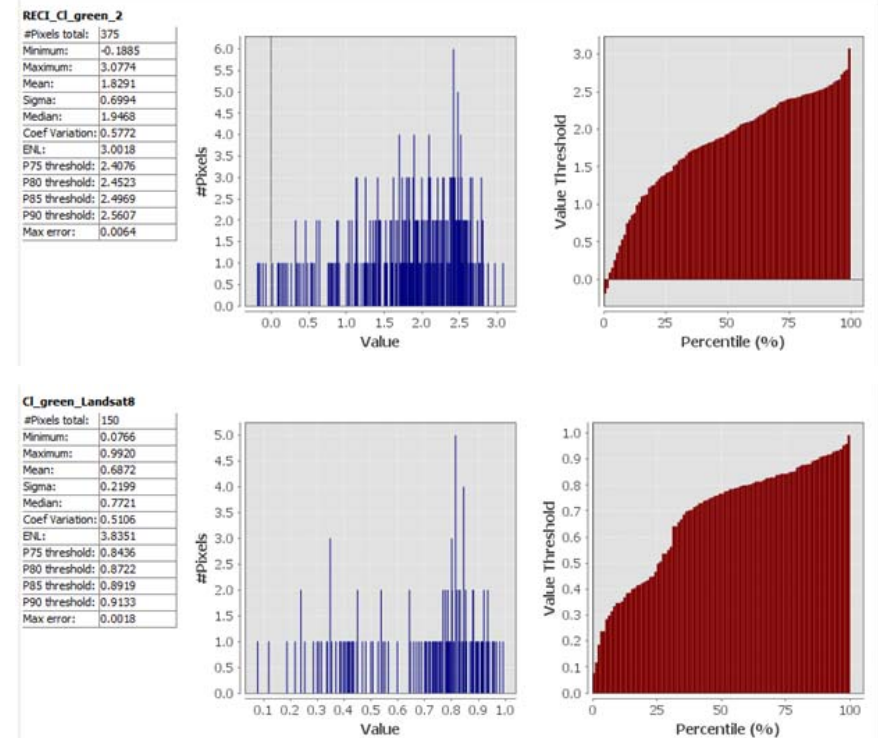

Figure 22. Statistics for Sentinel-2 (top) and Landsat-8 (bottom) Chlorophyll indexes.
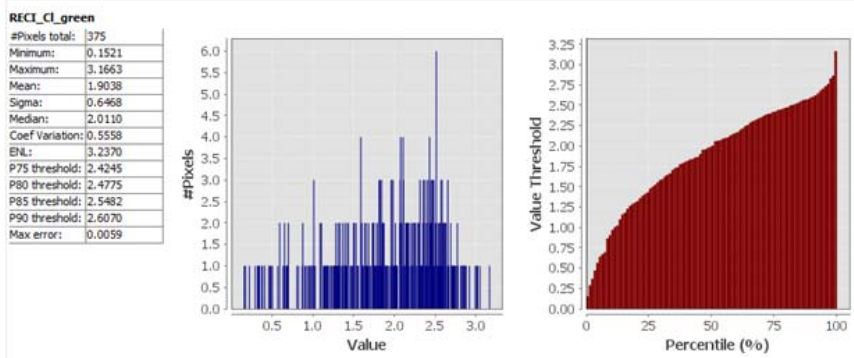

Figure 23. Statistics of RECl_Cl_Green index for Sentinel-2.

If a similar index, here named $\mathrm{Cl}$ _green_3, is generated for Sentinel- 2 by using the spectral Band $8 \mathrm{a}$ in place of the Band 7 , the (8) turns into (9):

$\mathrm{Cl}_{\text {Green }-} 3=\frac{\mathrm{B}_{8 \mathrm{a}}}{\mathrm{B}_{3}}-1$.

As reported in the statistics of Figure 24, the measured maximum values are even higher $(\max =3.743)$.

The band $8 \mathrm{a}$ in fact, is a band in a near infrared region that is not affected by water vapour and that appears to be sensitive to iron oxides present in plants or in the soil, unlike the other bands. See [31] for more details.

All the above results demonstrate how the use of Sentinel-2 data greatly enhances the information that can be obtained for the study of vegetation. Moreover since Sentinel-2 indexes provide the highest values, they are better to discriminate the
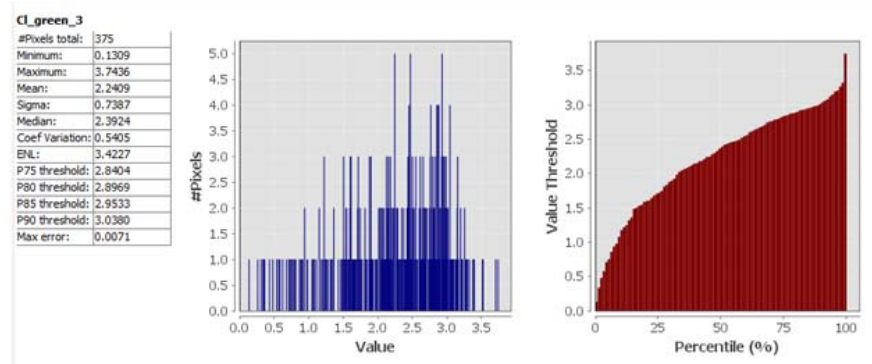

Figure 24. Statistics of Cl_green_3 for Sentinel-2. types of vegetation, because more marked differences are given.

At the beginning of this section we have discussed the comparison between Landsat-8 NDVI and Sentinel-1 CTest1 indexes by showing how the NDVI index can tell us how vegetation is: dry or healthy; and the CTest1 how geometry is.

Thanks to Sentinel-2 data availability a possible comparison can be done also between its NDVI index and Sentinel-1 CTest1, as shown in Figure 25.

The advantage is given by the Sentinel-2 resolution that allows to appreciate very small details, as already pointed out. Moreover all the new indexes, specific for vegetation analysis, can provide additional information on the type and status of vegetation by reaching to classify the coverage in a more appropriate way.

Our study based on different combinations of Landsat- 8 and Sentinel-2 bands, and on Sentinel-1 SAR images, through the calculation of so many different indexes such as, the NDVI and the CTest1 indexes, the Green Chlorophyll Index, the Red Edge Chlorophyll Index, and so on, has surely offered new tools for vegetation monitoring through the use of remote sensing techniques.

\section{CONCLUSIONS}

With the entry into operation of the Sentinel-2 mission in June 2015, a new land monitoring constellation of twin satellites has been added to Copernicus project from ESA and new insights have been derived through the combination of Sentinel-2 data with other optical/multispectral data, and with other data from satellites belonging to the same Copernicus project. To this end, the objective of this paper has been to present new added-value tools first through the integration of different satellite platforms: data from NASA Landsat- 8 and ESA Sentinel-1 have been used and combined, and furthermore through the comparison of satellite data from the same Copernicus project: data from Sentinel-1 and Sentinel-2 have been jointly processed and compared. Moreover data from
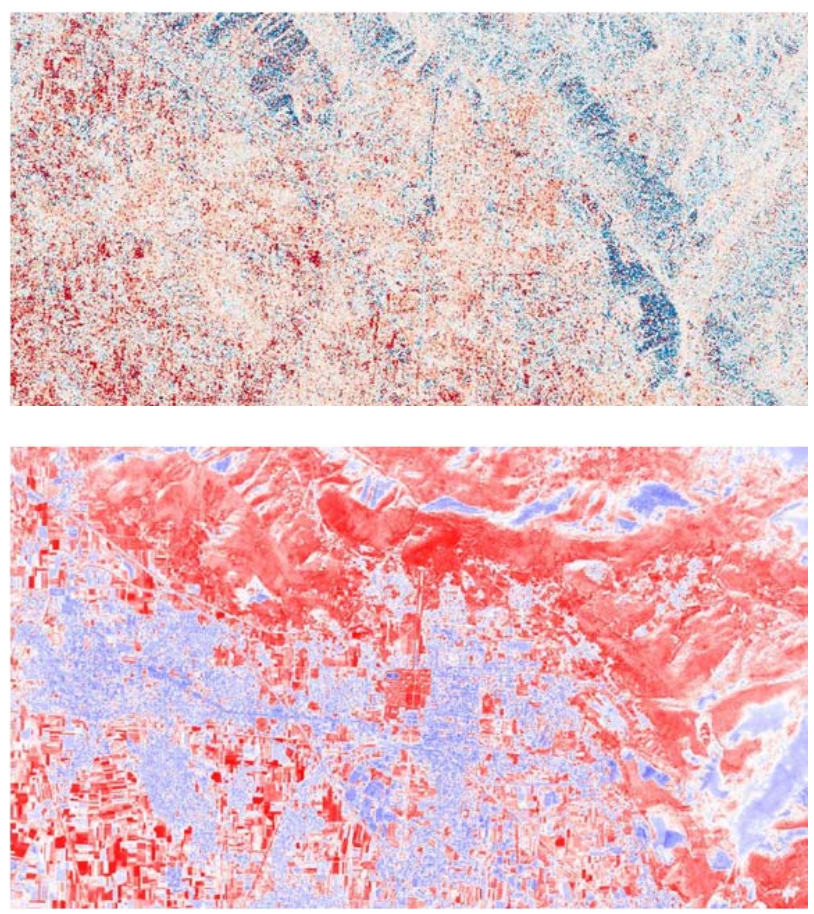

Figure 25. Sentinel-1 CTest1 (top) and Sentinel-2 NDVI (bottom). 
different optical/multispectral sensors, as those of Landsat- 8 and Sentinel-2, and data from SAR on board of Sentinel-1, have been all jointly analysed, compared and discussed by providing useful and interesting results. As future works it would be interesting to design a suitable "data fusion" algorithm to combine data and obtain information automatically on specific issues of interest.

At last, with respect to our previous research, the availability now of Sentinel-2 data has allowed very good improvements in terms of vegetation monitoring and definition, as obtained results have demonstrated.

\section{REFERENCES}

[1] Lillesand, T. and Kiefer R.W. and Chipman J. "Remote Sensing and Image Interpretation", Wiley and Sons, 2014, 7th Edition.

[2] Elachi, C. and vanZyl, J. "Introduction to the physics and techniques of remote sensing", Wiley and Sons, 2006, 2th Edition.

[3] Schott, J. R. " Remote Sensing. The Image Chain Approach", 2007, Oxford University Press.

[4] Masini, N. and Soldovieri, F. "Integrated non-invasive sensing techniques and geophysical methods for the study and conservation of architectural, archaeological and artistic heritage", Journal of Geophysics and Engineering, Vol.8, 2011.

[5] M. Focareta, S. Marcuccio, C. Votto, S.L. Ullo, "Combination of Landsat- 8 and Sentinel-1 data for the characterization of a site of interest. A Case Study: the Royal Palace of Caserta", 1rst International Conference on Metrology for Archaeology, Benevento, Italy, October 22-23, 2015.

[6] P.Addabbo, M.Focareta, S. Marcuccio, C. Votto, S.L. Ullo, "Land cover classification and monitoring through multisensory image and data combination", submitted to IGARSS'16, Beijing, China, July 10-15, 2016.

[7] http://landsat.gsfc.nasa.gov/landsat8

[8] F. De Zan,F. and Monti Guarnieri, A.M. "TOPSAR: Terrain Observation by Progressive Scans", IEEE Transactions on Geoscience and Remote Sensing, Vol.44, Issue 9, Sept.2006, pp. 2352-2360.

[9] Attema, E. "Mission Requirements Document for the European Radar Observatory Sentinel-1", ES-RS-ESA-SY-0007, Issue 1.4, 11 July 2005.

[10] Snoeij, P. and Attema, E. and Davidson, M. and Duesmann, B. and Floury, N. and Levrini, G. and Rommen, B. and Rosich, B. "The Sentinel-1 Radar Mission: Status and Performance, IEEE International Radar Conference-Surveillance for a Safer World, 12-16 Oct. 2009, Bordeaux, pp. 1-6

[11] Snoeij, P. and Attema, E. and Davidson, M. and Duesmann, B. and Floury, N. and Levrini, G. and Rommen, B. and Rosich, B. "The Sentinel-1 radar mission: status and performance", Proceedings of Radar Conference Surveillance for a Safer World, Oct. 2009, pp. 1-6.

[12] https://earth.esa.int/web/guest/missions/esa-operational-eomissions/sentinel-1.

[13] http://sarmap.ch.

[14] S. Cloude, E. Pottier "A Review of Target Decomposition Theorems in Radar Polarimetry", IEEE Transactions of Geoscience and Remote Sensing, Vol.34, No.2, pp. 498-518, March 1996.
[15] M. Ouarzeddine, and B. Souissi, "Unsupervised Classification Using Wishart Classifier”, USTHB, F.E.I, BP No. 32 EI Alia Bab Ezzouar, Alger.

http://earth.esa.int/workshops/polinsar2007/papers/227_ouarz eddine.pdf

[16] S. R. Cloude and E. Pottier, "An Entropy based classification scheme for land applications of polarimetric SAR," IEEE IGRS, vol.35, no.1, pp.68-78, Jan.1997.

[17] L. J. Du and J. S. Lee, "Polarimetric SAR image classification based on target decomposition theorem and complex Wishart distribution," Geoscience and Remote Sensing Symposium, 1996. IGARSS '96. 'Remote Sensing for a Sustainable Future.', International, Lincoln, NE, 1996, pp. 439-441 vol.1. http://ieeexplore.ieee.org/stamp/stamp.jsp?tp $=$ \&arnumber $=51$ 6366\&isnumber $=11016$

[18] J.S. Lee, M R Grunes, T L Ainsworth, L J Du, D L Schuler, S R Cloude, "Unsupervised Classification using Polarimetric Decomposition and Complex Wishart Distribution, " IEEE Transactions Geoscience and Remote Sensing, Vol 37/1, No. 5, pp2249-2259, Sep 1999.

[19] https://directory.eoportal.org/web/eoportal/satellitemissions/c-missions/copernicus-sentinel-2

[20] http://earthexplorer.usgs.gov/

[21] https://scihub.esa.int/

[22] http://www.realcasadiborbone.it/en/historydocuments/palaces-residences/the-royal-palace-of-caserta/

[23] Hornacek, M. and Wagner, W. and Sabel, D. and Hong-Linh Truong and Snoeij, P. and Hahmann, T. and Diedrich, E. and Doubkova, M. "Potential for High Resolution Systematic Global Surface Soil Moisture Retrieval via Change Detection Using Sentinel-1", IEEE Journal of Selected Topics in Applied Earth Observations and Remote Sensing, Vol.5, n.4, Aug. 2012, pp. 1303-1311.

[24] https://sentinel.esa.int/web/sentinel/sentinel-1-sar-wiki//wiki/Sentinel One/Algorithm+Overview

[25] J.G.P.W. Clevers, Anatoly Gitelson, publication/283419755, "Using the red-edge bands on Sentinel-2 for retrieving canopy chlophyll and nitrogen content", January 2012.

http://congrexprojects.com/docs/12c04_docs2/poster1_18_cle vers.pdf

[26] http://www.tankonyvtar.hu/en/tartalom/tamop425/0032_terin formatika/ch04s04.html

[27] Pinar, A. and Curran, P.J., "Grass Chlorophyll and the reflectance red edge", International Journal of Remote Sensing, 17, S. 351-357, (1996)

[28] Filella I. and Peñuelas, J., "The red edge position and shape as indicators of plant chlorophyll content, biomass and hydric status", International Journal of Remote Sensing 15, S.14591470, (1994).

[29] Weichelt, H. and Rosso, P. and Marx, A., and Reigber, S. and others, "The RapidEye Red Edge Band", white paper, http://www.blackbridge.com/rapideye/upload/Red_Edge_Whit e_Paper.pdf).

[30] Ilina B. Kamenova, "Opportunities of hyperspectral vegetation indices to assess nitrogen and chlorophyll content in crops", Technical report- Bulgarian Academy of Sciences, Space research and technology Institute Department of Remote Sensing and GIS, Sofia, Bulgaria.

http://csebr.cz/scerin2014/presentations/29_Kamenova.pdf

[31] Sentinel-2, User Handbook, ESA standard document, Issue 1, Rev 2, July 24, 2015. 